\title{
Stability of a Functional Equation Deriving from Quadratic and Additive Functions in Non-Archimedean Normed Spaces
}

\author{
Abasalt Bodaghi ${ }^{1}$ and Sang Og Kim ${ }^{2}$ \\ ${ }^{1}$ Department of Mathematics, Garmsar Branch, Islamic Azad University, Garmsar, Iran \\ ${ }^{2}$ Department of Mathematics, Hallym University, Chuncheon 200-702, Republic of Korea \\ Correspondence should be addressed to Abasalt Bodaghi; abasalt.bodaghi@gmail.com
}

Received 19 July 2013; Revised 27 August 2013; Accepted 7 September 2013

Academic Editor: Krzysztof Ciepliński

Copyright (C) 2013 A. Bodaghi and S. O. Kim. This is an open access article distributed under the Creative Commons Attribution License, which permits unrestricted use, distribution, and reproduction in any medium, provided the original work is properly cited.

We obtain the general solution of the generalized mixed additive and quadratic functional equation $f(x+m y)+f(x-m y)=$ $2 f(x)-2 m^{2} f(y)+m^{2} f(2 y), m$ is even; $f(x+y)+f(x-y)-2\left(m^{2}-1\right) f(y)+\left(m^{2}-1\right) f(2 y), m$ is odd, for a positive integer $m$. We establish the Hyers-Ulam stability for these functional equations in non-Archimedean normed spaces when $m$ is an even positive integer or $m=3$.

\section{Introduction}

The basic problem of the stability of functional equations was formulated by Ulam in 1940 in the following form. Suppose that a mapping $f$ satisfies the additive functional equation $f(x+y)=f(x)+f(y)$ only approximately. Then does there exist an additive function which approximates $f$ ? (See also [1].) In 1941, Hyers [2] gave the following answer to this question for Banach spaces. The result of Hyers was generalized in 1950 by Aoki [3] for approximately additive mappings and in 1978 by Rassias [4] for approximately linear mappings, by considering the unbounded Cauchy differences. A further generalization was obtained by Găvruţa [5] in 1994, by replacing the Cauchy differences by a control function $\varphi$ satisfying a very simple condition of convergence.

The Hyers-Ulam stability problem for the quadratic functional equation

$$
f(x+y)+f(x-y)=2 f(x)+2 f(y)
$$

was first proved by Skof for a function $f: \mathscr{X} \rightarrow \mathscr{Y}$, where $\mathscr{X}$ is a normed space and $\mathscr{Y}$ is a Banach space [6]. One year later, Cholewa [7] demonstrated that Skof's theorem is still true if relevant domain is replaced by an abelian group. After that, in [8], Czerwik proved the Hyers-Ulam stability of the quadratic functional equation (1) as a special case. In [9], it was shown that a mapping $f: \mathscr{X} \rightarrow \mathscr{Y}$ is quadratic if and only if $f(k x+$ $y)+f(k x-y)=2 k^{2} f(x)+2 f(y)$ for all $x, y \in \mathscr{X}$. Also, $f$ is quadratic if and only if $2 f((k x+k y) / 2)+2 f((k x-k y) / 2)=$ $k^{2} f(x)+k^{2} f(y)$ for all $x, y \in \mathscr{X}$ [10]. Cădariu and Radu investigated the stability of the Cauchy functional equation [11] and for the quadratic functional equation [12]. Stability problems of miscellaneous functional equations have been investigated by several authors during the last decades (see, e.g., $[13-15])$.

In [16], Eskandani et al. determined the general solution of the following mixed additive and quadratic functional equation:

$$
f(x+2 y)+f(x-2 y)+8 f(y)=2 f(x)+4 f(2 y) .
$$

They studied the Hyers-Ulam stability of (2) in nonArchimedean Banach modules over a unital Banach algebra. In [17], Najati and Moghimi established the general solution of the mixed type additive and quadratic functional equation

$$
\begin{aligned}
f(2 x & +y)+f(2 x-y) \\
& =f(x+y)+f(x-y)+2 f(2 x)-2 f(x)
\end{aligned}
$$

and investigated the stability of this equation in quasi-Banach spaces. The stability of (3) in random normed spaces is proved in [18]. 

tions:

In this paper, we consider the following functional equa-

$$
f(x+m y)+f(x-m y)=2 f(x)-2 m^{2} f(y)+m^{2} f(2 y),
$$

where $m$ is an even positive integer and

$$
\begin{aligned}
f(x+3 y)+f(x-3 y) & \\
& =f(x+y)+f(x-y)-16 f(y)+8 f(2 y) .
\end{aligned}
$$

Indeed, (4) and (5) are different from (2) and (3). It is easily verified that the function $f(x)=\alpha x^{2}+\beta x$ is a solution of the functional equations (4) and (5). We show that these functional equations are mixed additive and quadratic mappings. We also prove the Hyers-Ulam stability problem for these equations. As a corollary, the hyperstability of (4) and (5) under some conditions in non-Archimedean normed spaces is shown as well.

\section{General Solution of (4) and (5)}

To achieve our aim in this paper, we need the following lemma which is a fundamental tool.

Lemma 1. Let $\mathscr{X}$ and $\mathcal{Y}$ be real vector spaces.

(i) If an odd mapping $f: \mathscr{X} \rightarrow \mathcal{Y}$ satisfies the functional equation (4), then $f$ is additive.

(ii) If an odd mapping $f: X \rightarrow \mathcal{Y}$ satisfies the functional equation (5), then $f$ is additive.

(iii) If an even mapping $f: \mathscr{X} \rightarrow \mathcal{Y}$ satisfies the functional equation (4), then $f$ is quadratic.

(iv) If an even mapping $f: \mathscr{X} \rightarrow \mathscr{Y}$ satisfies the functional equation (5), then $f$ is quadratic.

Proof. (i) Letting $x=0$ in (4), we get $f(2 y)=2 f(y)$ for all $y \in \mathscr{X}$. This equality implies that

$$
f(x+m y)+f(x-m y)=2 f(x)
$$

for all $x, y \in \mathscr{X}$. Replacing $y$ by $y / m$ in (6), we have

$$
f(x+y)+f(x-y)=2 f(x)
$$

for all $x, y \in \mathscr{X}$. Substituting $x, y$ by $y, x$ in (7), respectively, we obtain

$$
f(x+y)-f(x-y)=2 f(y)
$$

for all $x, y \in \mathscr{X}$. The equalities (7) and (8) show that

$$
f(x+y)=f(x)+f(y) \quad(x, y \in \mathscr{X}) .
$$

(ii) Suppose that $f$ satisfies (5). Similar to the part (i), by the oddness of $f$, we have $f(2 x)=2 f(x)$ for all $x \in \mathscr{X}$. Thus

$$
f(x+3 y)+f(x-3 y)=f(x+y)+f(x-y)
$$

for all $x, y \in \mathscr{X}$. We substitute $x$ by $x+y$ in (10) and then $x$ by $x-y$ in (10); we get

$$
\begin{gathered}
f(x+4 y)+f(x-2 y)=f(x+2 y)+f(x), \\
f(x+2 y)+f(x-4 y)=f(x)+f(x-2 y)
\end{gathered}
$$

for all $x, y \in \mathscr{X}$. Then, by adding (11) to (12), we lead to

$$
f(x+4 y)+f(x-4 y)=2 f(x)
$$

for all $x, y \in \mathscr{X}$. Similar to the part (i), we can show that $f$ is additive.

(iii) By the assumption, the equality

$$
f(x+m y)+f(x-m y)=2 f(x)-2 m^{2} f(y)+m^{2} f(2 y)
$$

holds for a fixed even positive integer $m$. Putting $x=y=0$ in (14), we get $f(0)=0$. Once more, by letting $x=0$ in (14), we have

$$
2 f(m y)=-2 m^{2} f(y)+m^{2} f(2 y)
$$

for all $y \in \mathscr{X}$. Interchanging $x, y$ into $m x, x$ in (14), respectively, we deduce that

$$
f(2 m x)=2 f(m x)-2 m^{2} f(x)+m^{2} f(2 x)
$$

for all $x \in \mathscr{X}$. Plugging (15) into (16), we have $f(2 m x)=$ $4 f(m x)$ for all $x \in \mathscr{X}$ and thus $f(2 x)=4 f(x)$ for all $x \in \mathscr{X}$. Using the last equality and (14), we have

$$
f(x+m y)+f(x-m y)=2 f(x)+2 m^{2} f(y)
$$

for all $x, y \in \mathscr{X}$. Setting $x=0$ in (17), we obtain $f(m y)=$ $m^{2} f(y)$ for all $y \in \mathscr{X}$. Applying this equality and putting $x$ by $m x$ in (17), we get

$$
f(x+y)+f(x-y)=2 f(x)+2 f(y)
$$

for all $x, y \in \mathscr{X}$. This shows that $f$ is a quadratic mapping.

(iv) Suppose that $f$ satisfies (5). Replacing $x$ by $x+y$ and $x-y$ in (5), respectively, we have

$$
\begin{aligned}
f(x+ & 4 y)+f(x-2 y) \\
& =f(x+2 y)+f(x)-16 f(y)+8 f(2 y), \\
f(x+ & 2 y)+f(x-4 y) \\
& =f(x)+f(x-2 y)-16 f(y)+8 f(2 y)
\end{aligned}
$$

for all $x, y \in \mathscr{X}$. The equalities (19) and (20) imply that

$$
f(x+4 y)+f(x-4 y)=2 f(x)-32 f(y)+16 f(2 y)
$$

for all $x, y \in \mathscr{X}$. Now, the above equality is a special case of the part (iii) when $m=4$. 
In the following theorem, we solve (4) in which $m$ is an even positive integer and

$$
\begin{aligned}
& f(x+m y)+f(x-m y) \\
&= f(x+y)+f(x-y) \\
&-2\left(m^{2}-1\right) f(y)+\left(m^{2}-1\right) f(2 y),
\end{aligned}
$$

where $m$ is an odd positive integer.

Theorem 2. Let $X$ and $\mathscr{Y}$ be real vector spaces. Then $a$ mapping $f: X \rightarrow Y$ satisfies the functional equation (2) if and only if it satisfies

$$
\begin{aligned}
& f(x+m y)+f(x-m y) \\
& = \begin{cases}2 f(x)-2 m^{2} f(y)+m^{2} f(2 y), & m \text { is even, } \\
f(x+y)+f(x-y) & \\
-2\left(m^{2}-1\right) f(y)+\left(m^{2}-1\right) f(2 y), & m \text { is odd }\end{cases}
\end{aligned}
$$

for all $m \geq k$, where $k$ is a fixed positive integer with $k \geq 3$.

Proof. Suppose that $f: X \rightarrow \mathscr{Y}$ satisfies the functional equation (2). Putting $x=y=0$ in (2), we get $f(0)=0$. Replacing $x$ by $x+y$ and $x-y$ in (2), respectively, we have

$$
\begin{aligned}
f(x+3 y)+f(x-3 y) & \\
& =f(x+y)+f(x-y)-16 f(y)+8 f(2 y) .
\end{aligned}
$$

Similar to the above, we get

$$
f(x+4 y)+f(x-4 y)=2 f(x)-32 f(y)+16 f(2 y) .
$$

Using the above method, we can deduce that

$$
\begin{aligned}
& f(x+m y)+f(x-m y) \\
& =\left\{\begin{array}{cl}
2 f(x)-2 p_{m} f(y)+p_{m} f(2 y), & m \text { is even, } \\
f(x+y)+f(x-y) & \\
-2 p_{m} f(y)+p_{m} f(2 y), & m \text { is odd }
\end{array}\right.
\end{aligned}
$$

for all $x, y \in \mathscr{X}$ for which $p_{2}=4, p_{3}=8$ and

$$
p_{m}= \begin{cases}2 p_{m-1}-p_{m-2}+4 & m \text { is even, } \\ 2 p_{m-1}-p_{m-2} & m \text { is odd. }\end{cases}
$$

Solving the above recurrence equations, we get

$$
p_{m}= \begin{cases}m^{2} & m \text { is even, } \\ m^{2}-1 & m \text { is odd }\end{cases}
$$

for all positive integers $m \geq 2$.

Conversely, assume that $f: \mathscr{X} \rightarrow \mathscr{Y}$ satisfies the functional equations (4) and (22) for each $k \geq m$. Firstly, we assume that $m$ is even. For $k=m(m-1)$ and for each $x, y \in \mathscr{X}$, we have

$$
\begin{aligned}
f(x+ & m(m-1) y)+f(x-m(m-1) y) \\
& =2 f(x)-2 m^{2} f((m-1) y)+m^{2} f(2(m-1) y)
\end{aligned}
$$

for all $x, y \in \mathscr{X}$. On the other hand,

$$
\begin{aligned}
f(x & \left.+\left(m^{2}-m\right) y\right)+f\left(x-\left(m^{2}-m\right) y\right) \\
& =2 f(x)-2\left(m^{2}-m\right)^{2} f(y)+\left(m^{2}-m\right)^{2} f(2 y)
\end{aligned}
$$

for all $x, y \in \mathscr{X}$. It follows from (29) and (30) that

$$
\begin{aligned}
-2 f & ((m-1) y)+f(2(m-1) y) \\
& =-2(m-1)^{2} f(y)+(m-1)^{2} f(2 y)
\end{aligned}
$$

for all $x, y \in \mathscr{X}$. Since $(m+1)(m-1)$ is an odd number, we have

$$
\begin{aligned}
f(x+ & (m+1)(m-1) y)+f(x-(m+1)(m-1) y) \\
= & f(x+(m-1) y)+f(x-(m-1) y) \\
& -2\left((m+1)^{2}-1\right) f((m-1) y) \\
& +\left((m+1)^{2}-1\right) f(2(m-1) y)
\end{aligned}
$$

for all $x, y \in \mathscr{X}$. Also,

$$
\begin{aligned}
f(x+ & \left.\left(m^{2}-1\right) y\right)+f\left(x-\left(m^{2}-1\right) y\right) \\
= & f(x+y)+f(x-y)-2\left(\left(m^{2}-1\right)^{2}-1\right) f(y) \\
& +\left(\left(m^{2}-1\right)^{2}-1\right) f(2 y)
\end{aligned}
$$

for all $x, y \in \mathscr{X}$. Plugging (32) into (33) and using (31), we get

$$
\begin{aligned}
f(x+ & (m-1) y)+f(x-(m-1) y) \\
= & f(x+y)+f(x-y)-2\left((m-1)^{2}-1\right) f(y) \\
& +\left((m-1)^{2}-1\right) f(2 y) .
\end{aligned}
$$

For the odd case $m$, we have

$$
\begin{aligned}
f(x+ & (m+1)(m-1) y)+f(x-(m+1)(m-1) y) \\
= & 2 f(x)-2(m+1)^{2} f((m-1) y) \\
& +(m+1)^{2} f(2(m-1) y)
\end{aligned}
$$

for all $x, y \in \mathscr{X}$. Also

$$
\begin{aligned}
f(x & \left.+\left(m^{2}-1\right) y\right)+f\left(x-\left(m^{2}-1\right) y\right) \\
& =2 f(x)-2\left(m^{2}-1\right)^{2} f(y)+\left(m^{2}-1\right)^{2} f(2 y)
\end{aligned}
$$


for all $x, y \in \mathscr{X}$. The comparison of (35) and (36) shows that

$$
\begin{aligned}
-2 f & ((m-1) y)+f(2(m-1) y) \\
& =-2(m-1)^{2} f(y)+(m-1)^{2} f(2 y)
\end{aligned}
$$

for all $x, y \in \mathscr{X}$. For $k=m(m-1)$ and for each $x, y \in \mathscr{X}$, we have

$$
\begin{aligned}
& f(x+m(m-1) y)+f(x-m(m-1) y) \\
&= f(x+(m-1) y)+f(x-(m-1) y) \\
&-2\left(m^{2}-1\right) f((m-1) y)+\left(m^{2}-1\right) f(2(m-1) y)
\end{aligned}
$$

for all $x, y \in \mathscr{X}$. On the other hand,

$$
\begin{aligned}
f(x & \left.+\left(m^{2}-m\right) y\right)+f\left(x-\left(m^{2}-m\right) y\right) \\
& =2 f(x)-2\left(m^{2}-m\right)^{2} f(y)+\left(m^{2}-m\right)^{2} f(2 y)
\end{aligned}
$$

for all $x, y \in \mathscr{X}$. Now, by comparing (38) with (39) and applying (37), we obtain

$$
\begin{aligned}
f(x+ & (m-1) y)+f(x-(m-1) y) \\
& =2 f(x)-2(m-1)^{2} f(y)+(m-1)^{2} f(2 y)
\end{aligned}
$$

for all $x, y \in \mathscr{X}$. This completes the proof.

Theorem 3. Let $\mathscr{X}$ and $\mathcal{Y}$ be real vector spaces. A mapping $f: X \rightarrow \mathscr{Y}$ satisfies either (4) or (5) if and only if there exist a symmetric biadditive mapping $B: \mathscr{X} \times \mathscr{X} \rightarrow \mathscr{Y}$ and an additive mapping $A: \mathscr{X} \rightarrow \mathscr{Y}$ such that $f(x)=B(x, x)+$ $A(x)$ for all $x \in \mathscr{X}$.

Proof. Assume that there exist a symmetric biadditive mapping $B: \mathscr{X} \times \mathscr{X} \rightarrow \mathscr{Y}$ and an additive mapping $A: \mathscr{X} \rightarrow \mathscr{Y}$ such that $f(x)=B(x, x)+A(x)$ for all $x \in \mathscr{X}$. A simple computation shows that the mappings $A$ and $B^{\prime}: X \rightarrow Y$ given by $B^{\prime}(x)=B(x, x)$ satisfy the functional equations (4) and (5). Therefore the mapping $f$ satisfies (4) and (5).

Conversely, we decompose $f$ into the even part and odd part by setting

$$
f_{e}(x)=\frac{f(x)+f(-x)}{2}, \quad f_{o}(x)=\frac{f(x)-f(-x)}{2}
$$

for all $x \in \mathscr{X}$. Obviously, $f(x)=f_{e}(x)+f_{o}(x)$ for all $x \in \mathscr{X}$. One can easily check that the mappings $f_{e}$ and $f_{o}$ satisfy (4) and (5). It follows from Lemma 1 that the mappings $f_{e}$ and $f_{o}$ are quadratic and additive, respectively. Since $f_{e}$ is quadratic, by [19], there exists a symmetric biadditive mapping $B: \mathscr{X} \times$ $x \rightarrow \mathscr{Y}$ such that $f_{e}(x)=B(x, x)$ for all $x \in \mathscr{X}$. Thus $f(x)=$ $B(x, x)+A(x)$ for all $x \in \mathscr{X}$, where $A(x)=f_{o}(x)$ for all $x \in \mathscr{X}$.

\section{Hyers-Ulam Stability of (4) and (5)}

We recall some basic facts concerning non-Archimedean spaces and some preliminary results.

By a non-Archimedean field, we mean a field $\mathbb{K}$ equipped with a function (valuation) $|\cdot|$ from $\mathbb{K}$ into $[0, \infty)$ such that $|r|=0$ if and only if $r=0,|r s|=|r||s|$, and $|r+s| \leq$ $\max \{|r|,|s|\}$ for all $r, s \in \mathbb{K}$. Clearly $|1|=|-1|=1$ and $|n| \leq 1$ for all $n \in \mathbb{N}$.

Let $\mathscr{X}$ be a vector space over a scalar field $\mathbb{K}$ with a nonArchimedean nontrivial valuation $|\cdot|$. A function $\|\cdot\|: \mathscr{X} \rightarrow$ $\mathbb{R}$ is a non-Archimedean norm (valuation) if it satisfies the following conditions:

(i) $\|x\|=0$ if and only if $x=0$;

(ii) $\|r x\|=|r|\|x\|,(x \in \mathscr{X}, r \in \mathbb{K})$;

(iii) the strong triangle inequality (ultrametric), namely,

$$
\|x+y\| \leq \max \{\|x\|,\|y\|\} \quad(x, y \in \mathscr{X}) .
$$

Then $(\mathscr{X},\|\cdot\|)$ is called a non-Archimedean normed space. Due to the fact that

$$
\left\|x_{n}-x_{m}\right\| \leq \max \left\{\left\|x_{j+1}-x_{j}\right\| ; m \leq j \leq n-1\right\} \quad(n \geq m),
$$

a sequence $\left\{x_{n}\right\}$ is Cauchy if and only if $\left\{x_{n+1}-x_{n}\right\}$ converges to zero in a non-Archimedean normed space $\mathscr{X}$. By a complete non-Archimedean normed space, we mean one in which every Cauchy sequence is convergent.

In [20], Hensel discovered the $p$-adic numbers as a number theoretical analogue of power series in complex analysis. The most interesting example of non-Archimedean normed spaces is $p$-adic numbers. A key property of $p$-adic numbers is that they do not satisfy the Archimedean axiom; for all $x, y>0$, there exists an integer $n$ such that $x<n y$.

Let $p$ be a prime number. For any nonzero rational number $x=p^{r}(m / n)$ in which $m$ and $n$ are coprime to the prime number $p$. Consider the $p$-adic absolute value $|x|_{p}=p^{-r}$ on $\mathbb{Q}$. It is easy to check that $|\cdot|$ is a nonArchimedean norm on $\mathbb{Q}$. The completion of $\mathbb{Q}$ with respect to $|\cdot|$ which is denoted by $\mathbb{Q}_{p}$ is said to be the $p$-adic number field. One should remember that if $p>2$, then $\left|2^{n}\right|=1$ for all integers $n$. The stability of some functional equations in nonArchimedean spaces was investigated, for instance, in [21-24] (see also $[13,25])$.

Let $m$ be an even positive integer. We use the abbreviation for the given mapping $f: \mathscr{X} \rightarrow \mathscr{Y}$ as follows:

$$
\begin{aligned}
\mathscr{D}_{m} f(x, y):= & f(x+m y)+f(x-m y) \\
& -2 f(x)+2 m^{2} f(y)-m^{2} f(2 y), \\
\mathscr{D}_{3} f(x, y):= & f(x+3 y)+f(x-3 y)-f(x+y) \\
& -f(x-y)+16 f(y)-8 f(2 y) .
\end{aligned}
$$

From now on, we assume that $\mathscr{X}$ is a real vector space and $\mathcal{Y}$ is a complete non-Archimedean space unless otherwise stated explicitly. In the upcoming theorem, we prove the stability of functional equations (4) and (5). 
Theorem 4. Let $\phi: \mathscr{X} \times \mathscr{X} \rightarrow[0, \infty)$ be a function such that

$$
\lim _{k \rightarrow \infty} \frac{1}{|2|^{k}} \phi\left(2^{k} x, 2^{k} y\right)=0
$$

for all $x, y \in \mathscr{X}$. Suppose that $f: \mathscr{X} \rightarrow \mathscr{Y}$ is an odd mapping satisfying the inequality

$$
\left\|\mathscr{D}_{m} f(x, y)\right\| \leq \phi(x, y)
$$

for all $x, y \in \mathscr{X}$, where $m$ is an even positive integer or $m=3$. Then there exists a unique additive mapping $A: \mathscr{X} \rightarrow \mathscr{Y}$ such that

$$
\|f(x)-A(x)\| \leq \begin{cases}\frac{1}{\left|2 m^{2}\right|} \widetilde{\phi}(x), & m \text { is even, } \\ \frac{1}{|8|} \widetilde{\phi}(x), & m=3\end{cases}
$$

for all $x \in \mathscr{X}$, where $\widetilde{\phi}(x)=\sup \left\{\left(\phi\left(0,2^{j} x\right) /|2|^{j}\right): j \in \mathbb{N} \cup\{0\}\right\}$.

Proof. We prove the result when $m$ is even; another case is similar. Putting $x=0$ in (46), we have

$$
\|2 f(y)-f(2 y)\| \leq \frac{1}{|m|^{2}} \phi(0, y)
$$

for all $y \in \mathscr{X}$. Replacing $y$ by $2^{n} x$ in (48) and then dividing both sides by $|2|^{n+1}$, we get

$$
\left\|\frac{1}{2^{n}} f\left(2^{n} x\right)-\frac{1}{2^{n+1}} f\left(2^{n+1} x\right)\right\| \leq \frac{1}{|m|^{2}|2|^{n+1}} \phi\left(0,2^{n} x\right)
$$

for all $x \in \mathscr{X}$ and all nonnegative integers $n$. Thus the sequence $\left\{f\left(2^{n} x\right) / 2^{n}\right\}$ is Cauchy by (45) and (49). Completeness of the non-Archimedean space $y$ allows us to assume that there exists a mapping $A$, so that

$$
\lim _{n \rightarrow \infty} \frac{f\left(2^{n} x\right)}{2^{n}}=A(x) \text {. }
$$

For each $x \in \mathscr{X}$ and nonnegative integers $n$, we have

$$
\begin{aligned}
& \left\|\frac{f\left(2^{n} x\right)}{2^{n}}-f(x)\right\| \\
& \quad=\left\|\sum_{j=0}^{n-1} \frac{f\left(2^{j+1} x\right)}{2^{j+1}}-\frac{f\left(2^{j} x\right)}{2^{j}}\right\| \\
& \quad \leq \max \left\{\left\|\frac{f\left(2^{j+1} x\right)}{2^{j+1}}-\frac{f\left(2^{j} x\right)}{2^{j}}\right\|: 0 \leq j<n\right\} \\
& \quad \leq \frac{1}{\left|2 m^{2}\right|} \max \left\{\frac{\phi\left(0,2^{j} x\right)}{\mid 2^{j}}: 0 \leq j<n\right\} .
\end{aligned}
$$

Taking that $n$ tends to approach infinity in (51) and applying (50), we can see that inequality (47) holds when $m$ is even. It follows from (45), (46), and (50) that for all $x, y \in \mathscr{X}$,

$$
\begin{aligned}
\left\|\mathscr{D}_{m} A(x, y)\right\| & =\lim _{n \rightarrow \infty} \frac{1}{|2|^{n}}\left\|\mathscr{D}_{m} f\left(2^{n} x, 2^{n} y\right)\right\| \\
& \leq \lim _{n \rightarrow \infty} \frac{1}{|2|^{n}} \phi\left(2^{n} x, 2^{n} y\right)=0 .
\end{aligned}
$$

Hence, the mapping $A$ satisfies (4). Part (i) of Lemma 1 shows that the mapping $A$ is additive. Now, let $A^{\prime}: \mathscr{X} \rightarrow \mathscr{Y}$ be another additive mapping satisfying (47). Then we have

$$
\begin{aligned}
& \left\|A(x)-A^{\prime}(x)\right\| \\
& \quad=\lim _{k \rightarrow \infty} \frac{1}{|2|^{k}}\left\|A\left(2^{k} x\right)-A^{\prime}\left(2^{k} x\right)\right\| \\
& \leq \lim _{k \rightarrow \infty} \frac{1}{|2|^{k}} \max \left\{\left\|A\left(2^{k} x\right)-f\left(2^{k} x\right)\right\|,\right. \\
& \leq \frac{1}{\left|2 m^{2}\right|} \lim _{k \rightarrow \infty} \lim _{n \rightarrow \infty} \max \left\{\frac{\phi\left(0,2^{j} x\right)}{|2|^{j}}: k \leq j<n+k\right\} \\
& \quad=\frac{1}{\left|2 m^{2}\right|} \lim _{k \rightarrow \infty} \sup \left\{\frac{\left.\phi\left(2^{k} x\right)-A^{\prime}\left(2^{k} x\right) \|\right\}}{|2|^{j}}: k \leq j<\infty\right\}=0
\end{aligned}
$$

for all $x \in \mathscr{X}$. This shows the uniqueness of $A$.

We have the following result which is analogous to Theorem 4 for the functional equations (4) and (5). We include the proof for (4). The proof of (5) is similar.

Theorem 5. Let $\phi: \mathscr{X} \times \mathscr{X} \rightarrow[0, \infty)$ be a function such that

$$
\lim _{k \rightarrow \infty}|2|^{k} \phi\left(\frac{x}{2^{k}}, \frac{y}{2^{k}}\right)=0
$$

for all $x, y \in \mathscr{X}$. Suppose that $f: \mathscr{X} \rightarrow \mathcal{Y}$ is an odd mapping satisfying the inequality

$$
\left\|\mathscr{D}_{m} f(x, y)\right\|_{y} \leq \phi(x, y)
$$

for all $x, y \in \mathscr{X}$, where $m$ is an even positive integer or $m=3$. Then there exists a unique additive mapping $A: \mathscr{X} \rightarrow \mathscr{Y}$ such that

$$
\|f(x)-A(x)\| \leq \begin{cases}\frac{1}{|m|^{2}} \tilde{\phi}(x), & m \text { is even }, \\ \frac{1}{|8|} \tilde{\phi}(x), & m=3\end{cases}
$$

for all $x \in \mathscr{X}$, where $\widetilde{\phi}(x)=\sup \left\{|2|^{j} \phi\left(0,\left(x / 2^{j+1}\right)\right): j \in \mathbb{N} \cup\right.$ $\{0\}\}$.

Proof. We only obtain the result for the even integers. Similar to the proof of Theorem 4, we have

$$
\|2 f(y)-f(2 y)\| \leq \frac{1}{|m|^{2}} \phi(0, y)
$$

for all $y \in \mathscr{X}$. If we replace $y$ by $x / 2^{n+1}$ in inequality (57) and then multiply both sides of the result to $|2|^{n}$, we get

$$
\left\|2^{n+1} f\left(\frac{x}{2^{n+1}}\right)-2^{n} f\left(\frac{x}{2^{n}}\right)\right\| \leq \frac{|2|^{n}}{|m|^{2}} \phi\left(0, \frac{x}{2^{n+1}}\right)
$$


for all $x \in \mathscr{X}$ and all nonnegative integers $n$. Thus, we conclude from (54) and (58) that the sequence $\left\{2^{n} f\left(x / 2^{n}\right)\right\}$ is Cauchy. Since the non-Archimedean normed space $y$ is complete, this sequence converges in $\mathcal{Y}$ to the mapping $A$. Indeed,

$$
A(x)=\lim _{n \rightarrow \infty} 2^{n} f\left(\frac{x}{2^{n}}\right) \quad(x \in \mathscr{X})
$$

Using induction and (57), one can show that

$$
\begin{aligned}
& \left\|2^{n} f\left(\frac{x}{2^{n}}\right)-f(x)\right\| \\
& \quad \leq \frac{1}{|m|^{2}} \max \left\{|2|^{j} \phi\left(0, \frac{x}{2^{j+1}}\right): 0 \leq j<n\right\}
\end{aligned}
$$

for all $x \in \mathscr{X}$ and nonnegative integers $n$. Since the right-hand side of inequality (60) tends to be 0 as $n$ to approach infinity, by applying (59), we deduce inequality (56). Now, similar to the proof of Theorem 4, we can complete the rest of the proof.

Corollary 6. Let $\alpha, r$, and $s$ be positive real numbers such that $r, s \neq 1$ and $|2|<1$. Suppose that $\mathscr{X}$ is a non-Archimedean normed space and $f: \mathscr{X} \rightarrow \mathscr{Y}$ is an odd mapping fulfilling

$$
\left\|\mathscr{D}_{m} f(x, y)\right\| \leq \alpha\left(\|x\|^{r}+\|y\|^{s}\right)
$$

for all $x, y \in \mathscr{X}$, where $m$ is an even positive integer or $m=3$. Then there exists a unique additive mapping $A: \mathscr{X} \rightarrow \mathscr{Y}$ such that

$$
\|f(x)-A(x)\| \leq \begin{cases}\frac{\alpha\|x\|^{s}}{\left|2 m^{2}\right|} & m \text { is even, } r, s>1, \\ \frac{\alpha\|x\|^{s}}{|8|} & m=3, r, s>1, \\ \frac{\alpha\|x\|^{s}}{|m|^{2}|2|^{s}} & m \text { is even, } r, s<1, \\ \frac{\alpha\|x\|^{s}}{|8||2|^{s}} & m=3, r, s<1\end{cases}
$$

for all $x \in \mathscr{X}$.

Proof. The result follows from Theorems 4 and 5 by letting $\phi(x, y)=\alpha\left(\|x\|^{r}+\|y\|^{s}\right)$.

In the next result, we prove the hyperstability of the functional equations (4) and (5) under some conditions. Recall that a functional equation is called hyperstable if every approximate solution is an exact one (see, e.g., [13, 26-29]).

Corollary 7. Let $\alpha, r$, and $s$ be positive real numbers such that $r+s \neq 1$ and $|2|<1$. Suppose that $\mathscr{X}$ is a non-Archimedean

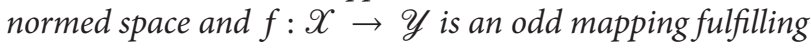

$$
\left\|\mathscr{D}_{m} f(x, y)\right\| \leq \alpha\|x\|^{r}\|y\|^{s}
$$

for all $x, y \in \mathscr{X}$, where $m$ is an even positive integer or $m=3$. Then $f$ is an additive mapping.
Proof. Taking $\phi(x, y)=\alpha\|x\|^{r}\|y\|^{s}$ in Theorems 4 and 5, we can obtain the desired result.

Theorem 8. Let $\phi: \mathscr{X} \times \mathscr{X} \rightarrow[0, \infty)$ be a function such that

$$
\lim _{k \rightarrow \infty} \frac{1}{|4|^{k}} \phi\left(2^{k} x, 2^{k} y\right)=0
$$

for all $x, y \in \mathscr{X}$. Suppose that $f: \mathscr{X} \rightarrow \mathcal{Y}$ is an even mapping satisfying the inequality

$$
\left\|\mathscr{D}_{m} f(x, y)\right\| \leq \phi(x, y)
$$

for all $x, y \in \mathscr{X}$, where $m$ is an even positive integer. Then there exists a unique quadratic mapping $Q: X \rightarrow \mathscr{Y}$ such that

$$
\|f(x)-Q(x)\| \leq \frac{1}{|4|} \widetilde{\phi}(x)
$$

for all $x \in \mathscr{X}$, where $\tilde{\phi}(x)=$ $\sup \left\{\left(\phi\left(0,\left(2^{j} x / m\right) /|4|^{j}\right),\left(\phi\left(2^{j} x,\left(2^{j} x / m\right)\right) /|4|^{j}\right): j \in \mathbb{N} \cup\{0\}\right\}\right.$.

Proof. It follows from (64) that $\phi(0,0)=0$. Thus (65) implies that $f(0)=0$. Putting $x=0$ in (65) and interchanging $y$ into $x$, we have

$$
\left\|2 f(m x)+2 m^{2} f(x)-m^{2} f(2 x)\right\| \leq \phi(0, x)
$$

for all $x \in \mathscr{X}$. Substituting $x, y$ by $m x, x$ in (65), respectively, we get

$$
\left\|f(2 m x)-2 f(m x)+2 m^{2} f(x)-m^{2} f(2 x)\right\| \leq \phi(m x, x)
$$

for all $x \in \mathscr{X}$. It follows from (67) and (68) that

$$
\|f(2 m x)-4 f(m x)\| \leq \max \{\phi(0, x), \phi(m x, x)\}
$$

for all $x \in \mathscr{X}$. Thus we have

$$
\|f(2 x)-4 f(x)\| \leq \max \left\{\phi\left(0, \frac{x}{m}\right), \phi\left(x, \frac{x}{m}\right)\right\}
$$

for all $x \in \mathscr{X}$. Replacing $x$ by $2^{n} x$ in (70) and then dividing both sides by $|4|^{n+1}$, we get

$$
\begin{aligned}
& \left\|\frac{1}{4^{n+1}} f\left(2^{n+1} x\right)-\frac{1}{4^{n}} f\left(2^{n} x\right)\right\| \\
& \quad \leq \frac{1}{|4|} \max \left\{\frac{\phi\left(0,\left(2^{n} x / m\right)\right)}{|4|^{n}}, \frac{\phi\left(2^{n} x,\left(2^{n} x / m\right)\right)}{|4|^{n}}\right\}
\end{aligned}
$$

for all $x \in \mathscr{X}$ and all nonnegative integers $n$. Thus the sequence $\left\{f\left(2^{n} x\right) / 4^{n}\right\}$ is Cauchy by (64) and (71). Since $y$ is complete, the sequence $\left\{f\left(2^{n} x\right) / 4^{n}\right\}$ converges in $y$ for all $x \in \mathscr{X}$. So one can define the mapping $Q: \mathscr{X} \rightarrow \mathscr{Y}$ by

$$
Q(x):=\lim _{n \rightarrow \infty} \frac{f\left(2^{n} x\right)}{4^{n}} \quad(x \in \mathscr{X}) .
$$


For each $x \in \mathscr{X}$ and nonnegative integers $n$, we have

$$
\begin{aligned}
& \left\|\frac{f\left(2^{n} x\right)}{4^{n}}-f(x)\right\| \\
& \quad=\left\|\sum_{j=0}^{n-1} \frac{f\left(2^{j+1} x\right)}{4^{j+1}}-\frac{f\left(2^{j} x\right)}{4^{j}}\right\| \\
& \quad \leq \max \left\{\left\|\frac{f\left(2^{j+1} x\right)}{4^{j+1}}-\frac{f\left(2^{j} x\right)}{4^{j}}\right\|: 0 \leq j<n\right\} \\
& \quad \leq \frac{1}{|4|} \max \left\{\frac{\phi\left(0,\left(2^{j} x / m\right)\right)}{|4|^{j}},\right. \\
& \left.\frac{\phi\left(2^{j} x,\left(2^{j} x / m\right)\right)}{|4|^{j}}: 0 \leq j<n\right\} .
\end{aligned}
$$

Taking $n$ to approach infinity in (73) and using (64) and (72), we find (66). Employing (64), (65), and (72), we obtain

$$
\begin{aligned}
\left\|\mathscr{D}_{m} Q(x, y)\right\| & =\lim _{n \rightarrow \infty} \frac{1}{|4|^{n}}\left\|\mathscr{D}_{m} f\left(2^{n} x, 2^{n} y\right)\right\| \\
& \leq \lim _{n \rightarrow \infty} \frac{1}{|4|^{n}} \phi\left(2^{n} x, 2^{n} y\right)=0 .
\end{aligned}
$$

Hence, the mapping $Q$ satisfies (4). It follows from part (iii) of Lemma 1 that the mapping $Q$ is quadratic. If $Q^{\prime}: \mathscr{X} \rightarrow \mathscr{Y}$ is another quadratic mapping satisfying (66), then

$$
\begin{aligned}
& \left\|Q(x)-Q^{\prime}(x)\right\| \\
& =\lim _{k \rightarrow \infty} \frac{1}{|4|^{k}}\left\|Q\left(2^{k} x\right)-Q^{\prime}\left(2^{k} x\right)\right\| \\
& \leq \lim _{k \rightarrow \infty} \frac{1}{|4|^{k}} \max \left\{\left\|Q\left(2^{k} x\right)-f\left(2^{k} x\right)\right\|,\right. \\
& \leq \frac{1}{|4|} \lim _{k \rightarrow \infty} \lim _{n \rightarrow \infty} \max \left\{\frac{\phi\left(0,\left(2^{j} x / m\right)\right)}{|4|^{j}},\right. \\
& \frac{1}{|4|} \lim _{k \rightarrow \infty} \sup \left\{\frac{\phi\left(2^{j} x,\left(2^{j} x / m\right)\right)}{|4|^{j}}: k \leq j<n+k\right\} \\
& \left.\frac{\phi\left(2^{j} x,\left(2^{j} x / m\right)\right)}{|4|^{j}}: k \leq j<\infty\right\}=0
\end{aligned}
$$

for all $x \in \mathscr{X}$. Therefore $Q=Q^{\prime}$. This completes the proof of the uniqueness of $Q$.
Theorem 9. Let $\phi: \mathscr{X} \times \mathscr{X} \rightarrow[0, \infty)$ be a function such that

$$
\lim _{k \rightarrow \infty}|4|^{k} \phi\left(\frac{x}{2^{k}}, \frac{y}{2^{k}}\right)=0
$$

for all $x, y \in \mathscr{X}$. Suppose that $f: \mathscr{X} \rightarrow \mathcal{Y}$ is an even mapping satisfying $f(0)=0$ and the inequality

$$
\left\|\mathscr{D}_{m} f(x, y)\right\| \leq \phi(x, y)
$$

for all $x, y \in \mathscr{X}$, where $m$ is an even positive integer. Then there exists a unique quadratic mapping $Q: X \rightarrow Y$ such that

$$
\|f(x)-Q(x)\| \leq \frac{1}{|4|} \widetilde{\phi}(x)
$$

for all $x \in \mathscr{X}$, where $\tilde{\phi}(x)=\sup \left\{|4|^{n} \phi\left(0,\left(x / 2^{n} m\right)\right)\right.$, $\left.|4|^{n} \phi\left(\left(x / 2^{n}\right),\left(x / 2^{n} m\right)\right): n \in \mathbb{N}\right\}$.

Proof. Similar to the proof of Theorem 8, we have

$$
\|f(2 x)-4 f(x)\| \leq \max \left\{\phi\left(0, \frac{x}{m}\right), \phi\left(x, \frac{x}{m}\right)\right\}
$$

for all $x \in \mathscr{X}$. Then we get

$$
\left\|f(x)-4 f\left(\frac{x}{2}\right)\right\| \leq \max \left\{\phi\left(0, \frac{x}{2 m}\right), \phi\left(\frac{x}{2}, \frac{x}{2 m}\right)\right\}
$$

for all $x \in \mathscr{X}$. Replacing $x$ by $x / 2^{n}$ in (80) and multiplying both sides to $|4|^{n}$, we get

$$
\begin{aligned}
& \left\|4^{n} f\left(\frac{x}{2^{n}}\right)-4^{n+1} f\left(\frac{x}{2^{n+1}}\right)\right\| \\
& \quad \leq \frac{1}{|4|} \max \left\{|4|^{n+1} \phi\left(0, \frac{x}{2^{n+1} m}\right),|4|^{n+1} \phi\left(\frac{x}{2^{n+1}}, \frac{x}{2^{n+1} m}\right)\right\}
\end{aligned}
$$

for all $x \in \mathscr{X}$ and all nonnegative integers $n$. Thus the sequence $\left\{4^{n} f\left(x / 2^{n}\right)\right\}$ is Cauchy by (76). The completeness of $\mathcal{Y}$ implies that the mentioned sequence is convergent. So we consider the mapping $Q: \mathscr{X} \rightarrow \mathscr{Y}$ by

$$
Q(x):=\lim _{n \rightarrow \infty} 4^{n} f\left(\frac{x}{2^{n}}\right) \quad(x \in \mathscr{X}) .
$$

For each $x \in \mathscr{X}$ and nonnegative integers $n$, we have

$$
\begin{aligned}
& \left\|f(x)-4^{n} f\left(\frac{x}{2^{n}}\right)\right\| \\
& \leq \frac{1}{|4|} \max \left\{|4|^{j} \phi\left(0, \frac{x}{2^{j} m}\right),|4|^{j} \phi\left(\frac{x}{2^{j}}, \frac{x}{2^{j} m}\right): 1 \leq j \leq n\right\} .
\end{aligned}
$$

Letting $n$ approach infinity in (83) and using (76) and (82), we can see that (78) holds. The rest of the proof is similar to the proof of Theorem 8 .

Corollary 10. Let $\alpha, r$, and $s$ be nonnegative real numbers and $|2|<1$. Suppose that $\mathscr{X}$ is a non-Archimedean normed space and $f: X \rightarrow Y$ is an even mapping fulfilling

$$
\left\|\mathscr{D}_{m} f(x, y)\right\| \leq \begin{cases}\alpha\|x\|^{r} & r \neq 2, \\ \alpha\left(\|x\|^{r}+\|y\|^{s}\right) & r, s \neq 2\end{cases}
$$


for all $x, y \in \mathscr{X}$, where $m$ is an even positive integer. Then there exists a unique quadratic mapping $Q: \mathscr{X} \rightarrow \mathscr{Y}$ such that

$$
\|f(x)-Q(x)\| \leq \begin{cases}\frac{\alpha\|x\|^{r}}{|4|} & r>2, s=0, \\ \frac{\alpha}{|4|}\left(\|x\|^{r}+\left\|\frac{x}{m}\right\|^{s}\right) & r, s>2, \\ \frac{\alpha\|x\|^{r}}{|2|^{r}} & r<2, s=0, \\ \alpha\left(\frac{\|x\|^{r}}{|2|^{r}}+\left\|\frac{x}{2 m}\right\|^{s}\right) & r, s<2\end{cases}
$$

for all $x \in \mathscr{X}$.

Theorem 11. Let $\phi: \mathscr{X} \times \mathscr{X} \rightarrow[0, \infty)$ be a function such that

$$
\lim _{k \rightarrow \infty} \frac{1}{|4|^{k}} \phi\left(2^{k} x, 2^{k} y\right)=0
$$

for all $x, y \in \mathscr{X}$. Suppose that $f: \mathscr{X} \rightarrow \mathcal{Y}$ is an even mapping satisfying the inequality

$$
\left\|\mathscr{D}_{3} f(x, y)\right\| \leq \phi(x, y)
$$

for all $x, y \in \mathscr{X}$. Then there exists a unique quadratic mapping $\mathrm{Q}: \mathcal{X} \rightarrow \mathcal{Y}$ such that

$$
\|f(x)-Q(x)\| \leq \frac{1}{|4|} \widetilde{\phi}(x)
$$

for all $x \in \mathscr{X}$, where $\widetilde{\phi}(x)=\sup \left\{|2|\left(\phi\left(\left(2^{j} x / 4\right),\left(2^{j} x / 4\right)\right) /|4|^{j}\right)\right.$, $\left(\phi\left((5 / 4) 2^{j} x,\left(2^{j} x / 4\right)\right) /|4|^{j}\right),\left(\phi\left((3 / 4) 2^{j} x,\left(2^{j} x / 4\right)\right) /|4|^{j}\right): j \in$ $\mathbb{N} \cup\{0\}\}$.

Proof. Similar to the proof of Theorem 8, we can show that $f(0)=0$. Replacing $x$ by $x+y$ and $x-y$ in (87), respectively, we get

$$
\begin{aligned}
& \| f(x+4 y)+f(x-2 y)-f(x+2 y) \\
& \quad-f(x)+16 f(y)-8 f(2 y) \| \leq \phi(x+y, y), \\
& \| f(x+2 y)+f(x-4 y)-f(x-2 y) \\
& \quad-f(x)+16 f(y)-8 f(2 y) \| \leq \phi(x-y, y)
\end{aligned}
$$

for all $x, y \in \mathscr{X}$. Inequalities (89) and (90) imply that

$$
\begin{gathered}
\|f(x+4 y)+f(x-4 y)-2 f(x)+32 f(y)-16 f(2 y)\| \\
\leq \max \{\phi(x+y, y), \phi(x-y, y)\}
\end{gathered}
$$

for all $x, y \in \mathscr{X}$. Interchanging $x, y$ into $4 x, x$ in (91), respectively, we obtain

$$
\begin{gathered}
\|f(8 x)-2 f(4 x)+32 f(x)-16 f(2 x)\| \\
\leq \max \{\phi(5 x, x), \phi(3 x, x)\}
\end{gathered}
$$

for all $x \in \mathscr{X}$. On the other hand, by putting $x=y$ in (87), we can deduce that

$$
\|f(4 x)-8 f(2 x)+16 f(x)\| \leq \phi(x, x)
$$

for all $x \in \mathscr{X}$. It follows from (92) and (93) that

$$
\|f(8 x)-4 f(4 x)\| \leq \max \{|2| \phi(x, x), \phi(5 x, x), \phi(3 x, x)\}
$$

for all $x \in \mathscr{X}$. Thus

$$
\begin{aligned}
& \left\|\frac{1}{4} f(2 x)-f(x)\right\| \\
& \quad \leq \frac{1}{|4|} \max \left\{|2| \phi\left(\frac{x}{4}, \frac{x}{4}\right), \phi\left(\frac{5}{4} x, \frac{x}{4}\right), \phi\left(\frac{3}{4} x, \frac{x}{4}\right)\right\}
\end{aligned}
$$

for all $x \in \mathscr{X}$. Substituting $x$ by $2^{n} x$ in (95) and then dividing both sides by $|4|^{n}$, we obtain

$$
\begin{aligned}
& \left\|\frac{1}{4^{n+1}} f\left(2^{n+1} x\right)-\frac{1}{4^{n}} f\left(2^{n} x\right)\right\| \\
& \leq \frac{1}{|4|} \max \left\{|2| \frac{\phi\left(\left(2^{n} x / 4\right),\left(2^{n} x / 4\right)\right)}{|4|^{n}},\right. \\
& \left.\frac{\phi\left((5 / 4) 2^{n} x,\left(2^{n} x / 4\right)\right)}{|4|^{n}}, \frac{\phi\left((3 / 4) 2^{n} x,\left(2^{n} x / 4\right)\right)}{|4|^{n}}\right\}
\end{aligned}
$$

for all $x \in \mathscr{X}$ and all nonnegative integers $n$. Thus the sequence $\left\{f\left(2^{n} x\right) / 4^{n}\right\}$ is Cauchy by (86) and (96). The completeness of $\mathscr{Y}$ implies that the sequence $\left\{f\left(2^{n} x\right) / 4^{n}\right\}$ is convergent. Define the mapping $Q: \mathscr{X} \rightarrow \mathscr{Y}$ via

$$
Q(x):=\lim _{n \rightarrow \infty} \frac{f\left(2^{n} x\right)}{4^{n}} \quad(x \in \mathscr{X}) .
$$

By a simple computation, one can show that

$$
\begin{gathered}
\left\|\frac{f\left(2^{n} x\right)}{4^{n}}-f(x)\right\| \\
\leq \frac{1}{|4|} \max \left\{|2| \frac{\phi\left(\left(2^{j} x / 4\right),\left(2^{j} x / 4\right)\right)}{|4|^{j}},\right. \\
\frac{\phi\left((5 / 4) 2^{j} x,\left(2^{j} x / 4\right)\right)}{|4|^{j}}, \\
\left.\frac{\phi\left((3 / 4) 2^{j} x,\left(2^{j} x / 4\right)\right)}{|4|^{j}}: 0 \leq j<n\right\}
\end{gathered}
$$

for all $x \in \mathscr{X}$ and for all $n \geq 0$. Taking $n$ to approach infinity in (98) and applying (86) and (97), we find (88). By (86), (87), and (97), we have

$$
\begin{aligned}
\left\|\mathscr{D}_{3} Q(x, y)\right\| & =\lim _{n \rightarrow \infty} \frac{1}{|4|^{n}}\left\|\mathscr{D}_{3} f\left(2^{n} x, 2^{n} y\right)\right\| \\
& \leq \lim _{n \rightarrow \infty} \frac{1}{|4|^{n}} \phi\left(2^{n} x, 2^{n} y\right)=0 .
\end{aligned}
$$


Hence, the mapping $Q$ satisfies (5). It follows from part (iv) of Lemma 1 that the mapping $Q$ is quadratic. Similar to the proof of Theorem 8 , one can show that $Q$ is unique.

Theorem 12. Let $\phi: \mathscr{X} \times \mathscr{X} \rightarrow[0, \infty)$ be a function such that

$$
\lim _{k \rightarrow \infty}|4|^{k} \phi\left(\frac{x}{2^{k}}, \frac{y}{2^{k}}\right)=0
$$

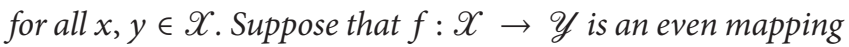
satisfying the inequality

$$
\left\|\mathscr{D}_{3} f(x, y)\right\| \leq \phi(x, y)
$$

for all $x, y \in \mathscr{X}$. Then there exists a unique quadratic mapping $Q: \mathscr{X} \rightarrow \mathcal{Y}$ such that

$$
\|f(x)-Q(x)\| \leq \frac{1}{|4|} \tilde{\phi}(x) \quad(x \in \mathscr{X})
$$

where $\widetilde{\phi}(x)=\sup \left\{|2||4|^{j} \phi\left(\left(x / 2^{j+3}\right),\left(x / 2^{j+3}\right)\right),|4|^{j} \phi\left(5\left(x / 2^{j+3}\right)\right.\right.$, $\left.\left.\left(x / 2^{j+3}\right)\right),|4|^{j} \phi\left(3\left(x / 2^{j+3}\right),\left(x / 2^{j+3}\right)\right): j \in \mathbb{N} \cup\{0\}\right\}$.

Proof. Similar to the proof of Theorem 8, one can obtain

$$
\|f(8 x)-4 f(4 x)\| \leq \max \{|2| \phi(x, x), \phi(5 x, x), \phi(3 x, x)\}
$$

for all $x \in \mathscr{X}$. Thus

$$
\begin{aligned}
\| f(x) & -4 f\left(\frac{x}{2}\right) \| \\
\leq & \max \left\{|2| \phi\left(\frac{x}{8}, \frac{x}{8}\right), \phi\left(\frac{5}{8} x, \frac{x}{8}\right), \phi\left(\frac{3}{8} x, \frac{x}{8}\right)\right\}
\end{aligned}
$$

for all $x \in \mathscr{X}$. Substituting $x$ by $x / 2^{n}$ in (104) and then multiplying both sides to $|4|^{n}$, we have

$$
\begin{aligned}
& \left\|4^{n} f\left(\frac{x}{2^{n}}\right)-4^{n+1} f\left(\frac{x}{2^{n+1}}\right)\right\| \\
& \leq \max \left\{|2||4|^{n} \phi\left(\frac{x}{2^{n+3}}, \frac{x}{2^{n+3}}\right),\right. \\
& \left.|4|^{n} \phi\left(5 \frac{x}{2^{n+3}}, \frac{x}{2^{n+3}}\right),|4|^{n} \phi\left(3 \frac{x}{2^{n+3}}, \frac{x}{2^{n+3}}\right)\right\}
\end{aligned}
$$

for all $x \in \mathscr{X}$ and all nonnegative integers $n$. The last inequality and (100) imply that the sequence $\left\{f\left(2^{n} x\right) / 4^{n}\right\}$ is Cauchy. So, this sequence converges to the mapping $Q: \mathscr{X} \rightarrow$ $\mathcal{y}$. In other words,

$$
Q(x):=\lim _{n \rightarrow \infty} \frac{f\left(2^{n} x\right)}{4^{n}} \quad(x \in \mathscr{X}) .
$$

We can also show that

$$
\begin{gathered}
\left\|f(x)-4^{n} f\left(\frac{x}{2^{n}}\right)\right\| \\
\leq \frac{1}{|4|} \max \left\{|2||4|^{j} \phi\left(\frac{x}{2^{j+3}}, \frac{x}{2^{j+3}}\right),|4|^{j} \phi\left(5 \frac{x}{2^{j+3}}, \frac{x}{2^{j+3}}\right),\right. \\
\left.|4|^{j} \phi\left(3 \frac{x}{2^{j+3}}, \frac{x}{2^{j+3}}\right): 0 \leq j<n\right\}
\end{gathered}
$$

for all $x \in \mathscr{X}$ and for all $n \geq 0$. Taking $n$ to approach infinity in (107) and applying (100) and (101), we see that inequality (102) holds. Now, similar to the proof of Theorem 8 , one can show that $Q$ is a unique quadratic mapping.

Corollary 13. Let $\alpha$ and $r$ be positive real numbers such that $r \neq 2$ and $|2|<1$. Suppose that $\mathscr{X}$ is a non-Archimedean

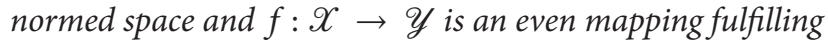

$$
\left\|\mathscr{D}_{3} f(x, y)\right\| \leq \alpha\|y\|^{r}
$$

for all $x, y \in \mathscr{X}$. Then there exists a unique quadratic mapping $\mathrm{Q}: \mathscr{X} \rightarrow \mathcal{Y}$ such that

$$
\|f(x)-Q(x)\| \leq \begin{cases}\frac{|2|\|x\|^{r}}{|4|^{r+1}} \alpha & r>2, \\ \frac{\|x\|^{r}}{|2||8|^{r}} \alpha & r<2\end{cases}
$$

for all $x \in \mathscr{X}$.

Proof. Letting $\phi(x, y)=\alpha\|y\|^{r}$ in Theorems 11 and 12, one can obtain the required result.

\section{Conflict of Interests}

The authors declare that there is no conflict of interests regarding the publication of this paper.

\section{Acknowledgments}

The authors express their sincere thanks to the reviewers for the careful and detailed reading of the paper and very helpful suggestions that improved the paper substantially.

\section{References}

[1] S. M. Ulam, Problems in Modern Mathematics, Science Editions, chapter 6, John Wiley \& Sons, New York, NY, USA, 1940.

[2] D. H. Hyers, "On the stability of the linear functional equation," Proceedings of the National Academy of Sciences of the United States of America, vol. 27, pp. 222-224, 1941.

[3] T. Aoki, "On the stability of the linear transformation in Banach spaces," Journal of the Mathematical Society of Japan, vol. 2, pp. 64-66, 1950.

[4] T. M. Rassias, "On the stability of the linear mapping in Banach spaces," Proceedings of the American Mathematical Society, vol. 72, no. 2, pp. 297-300, 1978.

[5] P. Găvruţa, "A generalization of the Hyers-Ulam-Rassias stability of approximately additive mappings," Journal of Mathematical Analysis and Applications, vol. 184, no. 3, pp. 431-436, 1994.

[6] F. Skof, "Local properties and approximation of operators," Rendiconti del Seminario Matematico e Fisico di Milano, vol. 53, pp. 113-129, 1983.

[7] P. W. Cholewa, "Remarks on the stability of functional equations," Aequationes Mathematicae, vol. 27, no. 1-2, pp. 76-86, 1984.

[8] St. Czerwik, "On the stability of the quadratic mapping in normed spaces," Abhandlungen aus dem Mathematischen Seminar der Universität Hamburg, vol. 62, pp. 59-64, 1992. 
[9] J. R. Lee, J. S. An, and C. Park, "On the stability of quadratic functional equations," Abstract and Applied Analysis, vol. 2008, Article ID 628178, 8 pages, 2008.

[10] M. E. Gordji and A. Bodaghi, "On the Hyers-Ulam-Rassias stability problem for quadratic functional equations," East Journal on Approximations, vol. 16, no. 2, pp. 123-130, 2010.

[11] L. Cădariu and V. Radu, "On the stability of the Cauchy functional equation: a fixed point approach," in Iteration theory (ECIT '02), vol. 346 of Grazer Mathematische Berichte, pp. 4352, Karl-Franzens-Universität Graz, Graz, Austria, 2004.

[12] L. Cădariu and V. Radu, "Fixed points and the stability of quadratic functional equations," Analele Universităţii de Vest din Timişoara, Seria Matematică-Informatică, vol. 41, no. 1, pp. 2548, 2003.

[13] N. Brillouët-Belluot, J. Brzdęk, and K. Ciepliński, "On some recent developments in Ulam's type stability," Abstract and Applied Analysis, vol. 2012, Article ID 716936, 41 pages, 2012.

[14] K. Ciepliński, "Applications of fixed point theorems to the Hyers-Ulam stability of functional equations-a survey," Annals of Functional Analysis, vol. 3, no. 1, pp. 151-164, 2012.

[15] S. M. Jung, Hyers-Ulam-Rassias Stability of Functional Equations in Nonlinear Analysis, vol. 48 of Springer Optimization and Its Applications, Springer, New York, NY, USA, 2011.

[16] G. Z. Eskandani, H. Vaezi, and Y. N. Dehghan, "Stability of a mixed additive and quadratic functional equation in nonArchimedean Banach modules," Taiwanese Journal of Mathematics, vol. 14, no. 4, pp. 1309-1324, 2010.

[17] A. Najati and M. B. Moghimi, "Stability of a functional equation deriving from quadratic and additive functions in quasi-Banach spaces," Journal of Mathematical Analysis and Applications, vol. 337, no. 1, pp. 399-415, 2008.

[18] S. S. Jin and Y. H. Lee, "On the stability of the functional equation deriving from quadratic and additive function in random normed spaces via fixed point method," Journal of the Chungcheong Mathematical Society, vol. 25, no. 1, pp. 51-63, 2012.

[19] J. Aczel and J. Dhombres, Functional Equations in Several Variables, vol. 31 of Encyclopedia of Mathematics and Its Applications, Cambridge University Press, Cambridge, UK, 1989.

[20] K. Hensel, "Uber eine neue Begrndung der Theorie der algebraischen Zahlen," Jahresbericht der Deutschen MathematikerVereinigung, vol. 6, no. 3, pp. 83-88, 1897.

[21] L. M. Arriola and W. A. Beyer, "Stability of the Cauchy functional equation over $p$-adic fields," Real Analysis Exchange, vol. 31, no. 1, pp. 125-132, 2005.

[22] J. Brzdęk and K. Ciepliński, "A fixed point approach to the stability of functional equations in non-Archimedean metric spaces," Nonlinear Analysis: Theory, Methods \& Applications, vol. 74, no. 18, pp. 6861-6867, 2011.

[23] M. S. Moslehian and T. M. Rassias, "Stability of functional equations in non-Archimedean spaces," Applicable Analysis and Discrete Mathematics, vol. 1, no. 2, pp. 325-334, 2007.

[24] J. Schwaiger, "Functional equations for homogeneous polynomials arising from multilinear mappings and their stability," Annales Mathematicae Silesianae, no. 8, pp. 157-171, 1994.

[25] T. Z. Xu, "Stability of multi-Jensen mappings in nonArchimedean normed spaces," Journal of Mathematical Physics, vol. 53, no. 2, Article ID 023507, 9 pages, 2012.

[26] A. Bodaghi, I. A. Alias, and M. H. Ghahramani, "Ulam stability of a quartic functional equation," Abstract and Applied Analysis, vol. 2012, Article ID 232630, 9 pages, 2012.
[27] J. Brzdęk, "Stability of the equation of the $p$-Wright affine functions," Aequationes Mathematicae, vol. 85, no. 3, pp. 497503, 2013.

[28] G. Maksa and Z. Páles, "Hyperstability of a class of linear functional equations," Acta Mathematica, vol. 17, no. 2, pp. 107$112,2001$.

[29] M. Piszczek and J. Szczawińska, "Hyperstability of the drygas functional equation," Journal of Function Spaces and Applications, vol. 2013, Article ID 912718, 4 pages, 2013. 


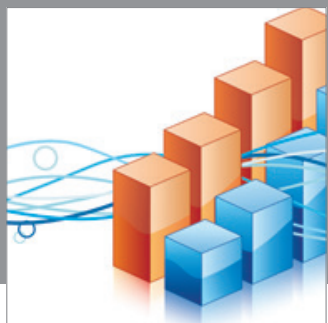

Advances in

Operations Research

mansans

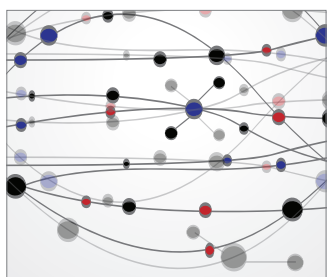

The Scientific World Journal
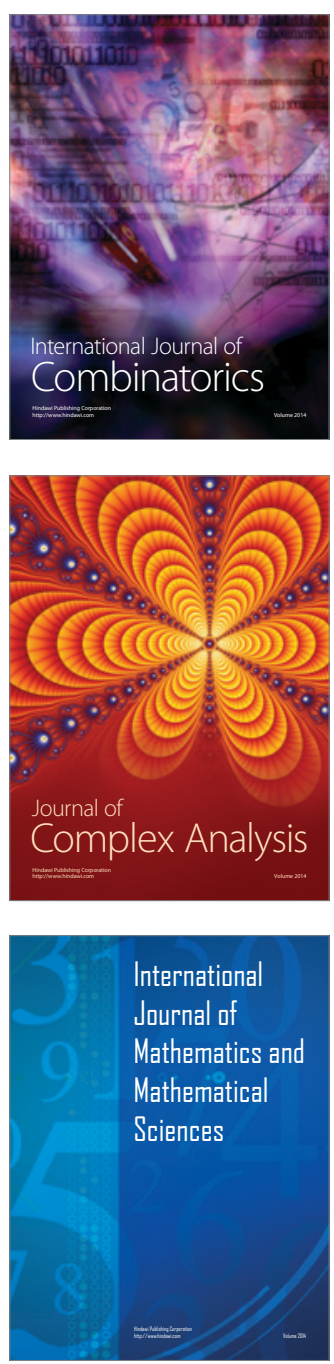
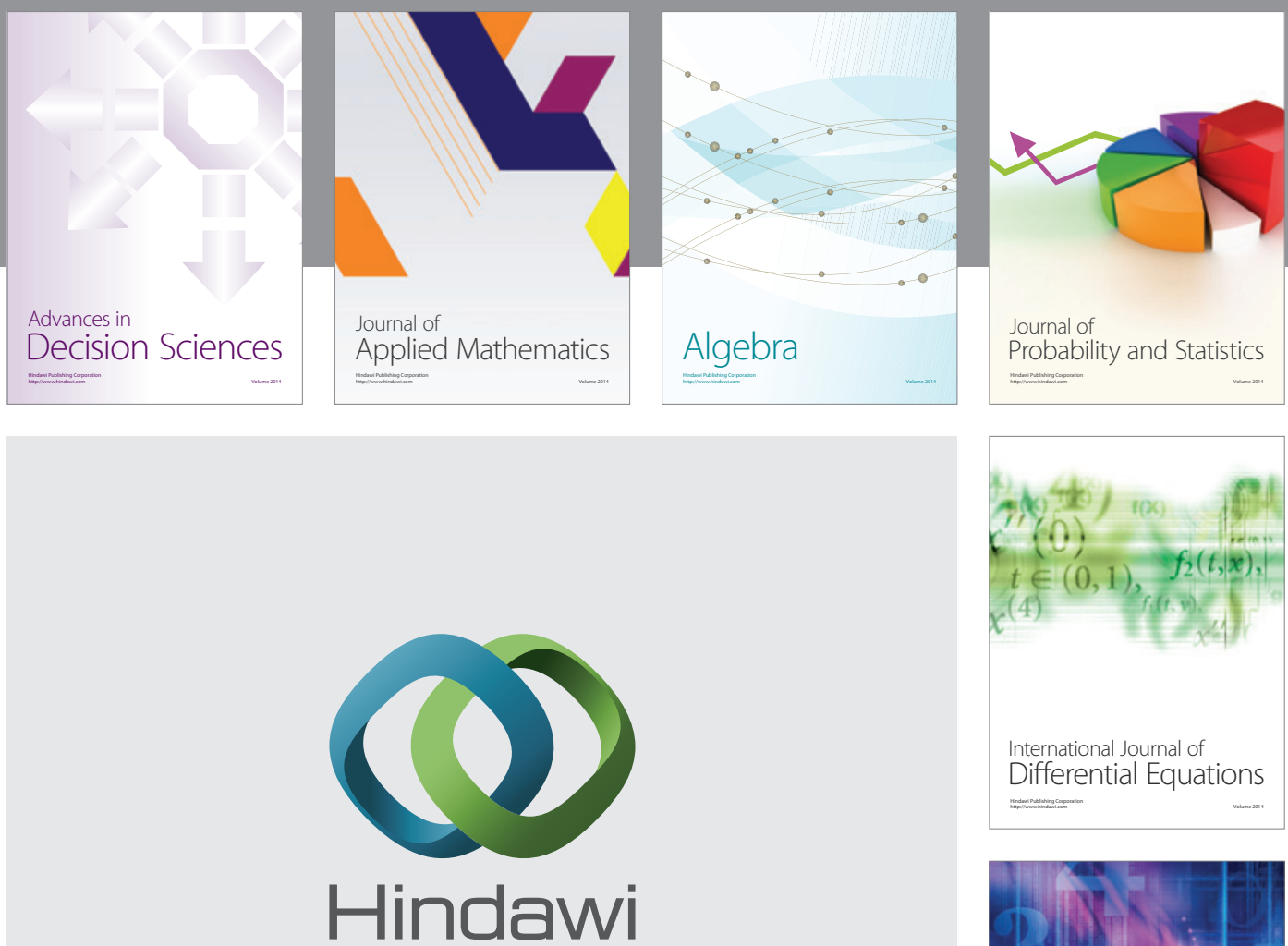

Submit your manuscripts at http://www.hindawi.com
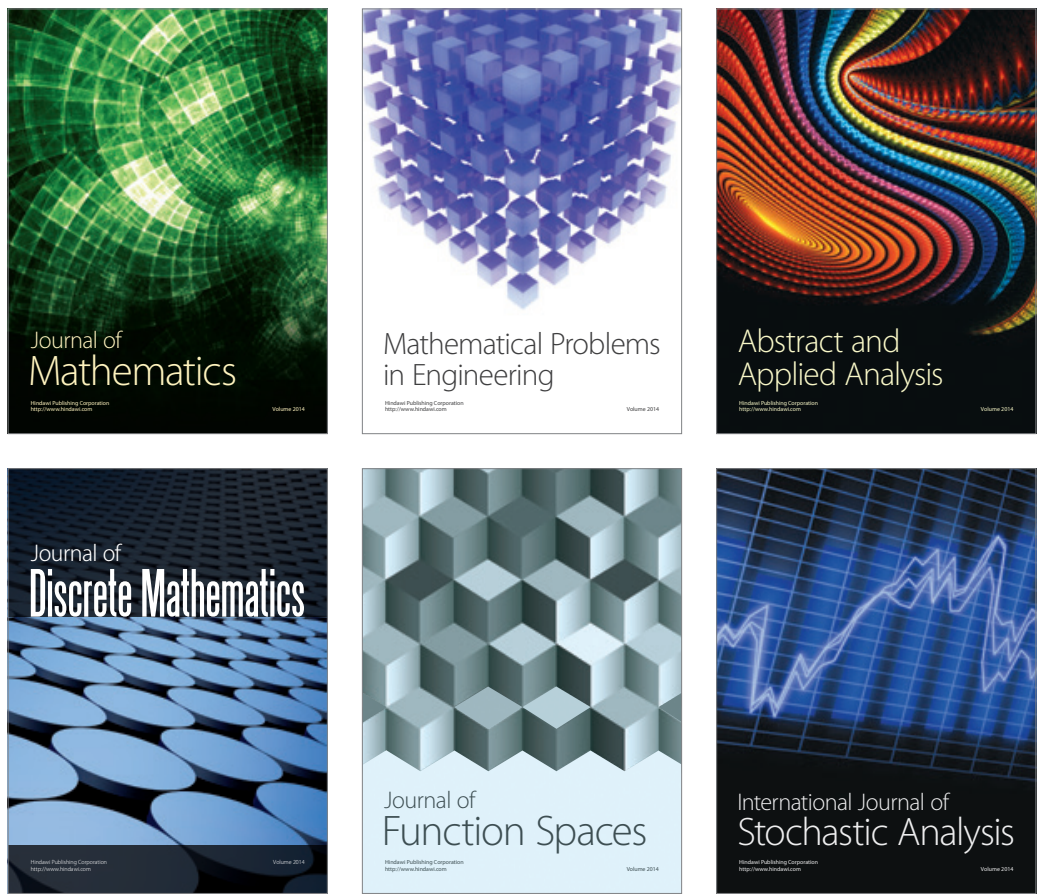

Journal of

Function Spaces

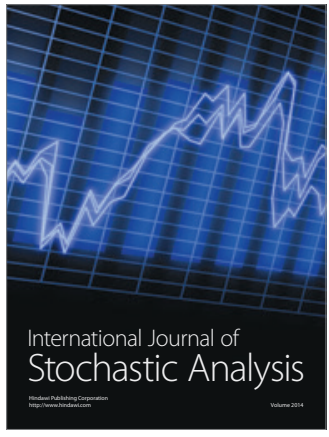

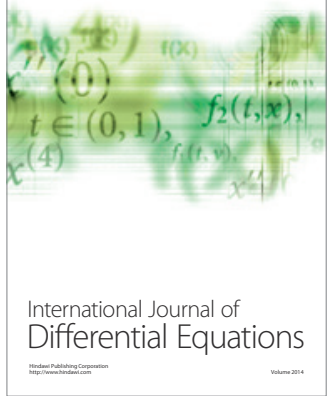
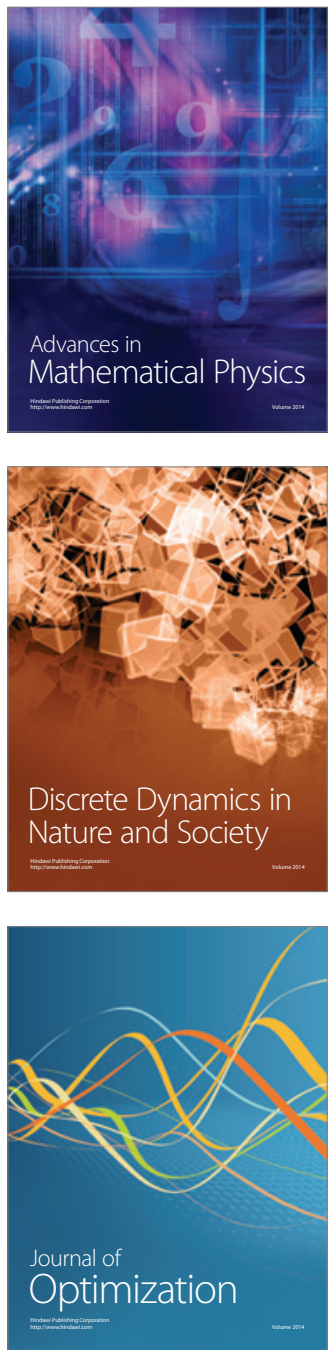